\title{
The Quantization of General Relativity: Photon Mediates Gravitation
}

\author{
Azzam AlMosallami
}

\begin{abstract}
In this paper I propose a quantization of general relativity of Einstein which leads to photon mediates gravitation. My quantization of GR depends on the modified special relativity MSRT which introduces a new interpretation to the Lorentz transformation equations depending on quantum theory (Copenhagen school) [25, 26, 27, 43]. My new interpretation to the Lorentz transformation leads to the Lorentz transformation is vacuum energy dependent instead of the relative velocity in Einstein's interpretation to the Lorentz transformation equations in the SRT. Furthermore the Lorentz factor is equivalent to the refractive index in optics. In my interpretation to the Lorentz transformation I refuse the reciprocity principle which was adopted by Einstein in the SRT. Refusing the reciprocity principle in my theory leads to disappearing all the paradoxes in the SRT; the Twin paradox, Ehrenfest paradox, Ladder paradox and Bell's spaceship paradox. Furthermore, according to my interpretation I could reconcile and interpret the experimental results of quantum tunneling and entanglement (spooky action), —Casimir effect, Hartman effect- with the SRT in this paper. My new interpretation leads also to the wave-particle duality as in quantum theory, and thus agrees with Heisenberg uncertainty principle. The generalization of my transformation leads also the concept of acceleration or deceleration is vacuum fluctuations as in quantum field theory. In my proposed quantized force, the force is given as a function of frequency [1]. Where, in this paper I defined the relativistic momentum as a function of frequency equivalent to the relativistic kinetic energy held by a body and time, and then the quantized force is given as the first derivative of the momentum with respect to time. Subsequently I introduce Newton's second law as it is relativistic quantized force, and then I introduce the relativistic quantized inertial force, and then by my equivalence principle which agreed completely with the experimental results of QFT, I introduce the relativistic quantized gravitational force, and the quantized gravitational time dilation.
\end{abstract}

Index Terms - Special relativity, general relativity, Lorentz transformation equations, faster than light, wormholes. Pioneer anomaly.

\section{THE QuANTIZATION OF SPECIAL RELATIVITY}

\section{INTRODUCTION}

$\mathrm{M}$ Y paper (The modified special relativity theory MSRT) [27] is considered as a new understanding to the Lorentz transformation equations depending on the concepts of quantum theory (Copenhagen School). It is a new formulation to the time dilation, length contraction and the speed of light which are vacuum energy dependent. What I proposed in my modified Lorentz transformation is agreed and interpreting the experimental results of quantum tunneling (Gunter Nimtz experiments) and quantum entanglement. Furthermore, it is disappeared all the paradoxes in SRT. Recently, there are some voices in physics asking for the variability of the speed of light, one of them the Portuguese cosmologist and professor in Theoretical Physics at Imperial College London João Magueijo. In 1998, Magueijo teamed with Andreas Albrecht to work on the varying speed of light (VSL) theory of cosmology, which proposes that the speed of light was much higher in the early universe, of 60 orders of magnitude faster than its present value. This would explain the horizon problem (since distant regions of the expanding universe would have had time to interact and homogenize their properties), and is presented as an alternative to the more mainstream theory of cosmic inflation [36]. My new interpretation of the Lorentz transformation equations reconciles and interprets the variability of the speed of light in SRT which is vacuum energy dependent. Also

\footnotetext{
- Azzam AlMosallami is currently The Director of the Science Center for Studies and Research, Switzerland, PH-+41791206234 Email: a.almosallami71@gmail.com
}

recently, two published papers in European Physical Journal D challenge established wisdom about the nature of vacuum. In one paper, Marcel Urban from the University of Paris-Sud, located in Orsay, France and his colleagues identified a quantum level mechanism for interpreting vacuum as being filled with pairs of virtual particles with fluctuating energy values. As a result, the inherent characteristics of vacuum, like the speed of light, may not be a constant after all, but fluctuate [35]. Meanwhile, in another study, Gerd Leuchs and Luis L. Sánchez-Soto, from the Max Planck Institute for the Physics of Light in Erlangen, Germany, suggest that physical constants, such as the speed of light and the so-called impedance of free space, are indications of the total number of elementary particles in nature [39]. Also, two separate research groups, one of which is from MIT, have presented evidence that wormholes - tunnels that may allow us to travel through time and space - are "powered" by quantum entanglement. Furthermore, one of the research groups also postulates the reverse - that quantum entangled particles are connected by miniature wormholes. These ideas are agreed and predicted in my paper $[33,34]$.

The dependency of the speed of light on the vacuum energy is adopted in my interpretation to the Lorentz transformation equations, which is the lost key of unifying between quantum theory and relativity (special and general).

\section{The Theory}


In my Modified Special relativity (MSRT)[27], and my new interpretation to the Lorentz transformation equations, I found, when the train is moving with constant speed $v$, its vacuum energy is increased compared to the vacuum energy of the earth surface. And when the light beam is passing through the vacuum of the moving train, it is equivalent to passing through a medium of refractive index greater than 1 . In this case, I found in my MSRT, the time required for the light beam to pass the length of the moving train for the earth observer is independent of the direction of the velocity of the train compared to the direction of transmitting the light beam [37]. Thus, if the light beam is sent inside the moving train from the back to the front -at the direction of the velocity- in this case for the earth observer according to his clock the required time separation for the light beam to pass the length of the moving train is $\Delta t$ where

$$
\Delta t=\frac{L}{\sqrt{c^{2}-v^{2}}}
$$

Also if the light beam is sent from the front of the moving train to the back at the opposite direction of the direction of the velocity of the train, then the measured time separation for the light beam to pass the length of the moving train for the earth observer according to his earth clock is also given according to eq. (1). From eq. (1), the measured speed of light inside the moving train for the stationary earth observer according to his earth clock is $c^{\prime}$ where

$$
c^{\prime}=\sqrt{c^{2}-v^{2}}
$$

Where $c^{\prime}$ does not depend on the direction of transmitting the light beam compared to the direction of the velocity of the train. It depends only on the absolute value of the velocity of the train. This proposed solution - the independency of the measured speed of light inside the moving frame with the direction of the velocity of the moving frame - explains the negative result of the Michelson-Morley experiment [34] as we shall see later in my new interpretation of the Lorentz transformation equations.

In my MSRT I proposed also, the length of the moving train

$L$ is the same if the train was stationary for the stationary earth observer, where I refute the length contraction in the special relativity of Einstein that the length of the moving frame will be contracted in the direction of the velocity for the earth observer. From that we get, when the train is stationary, and a light beam is sent along its length, we get

$$
L=c \Delta t_{0}
$$

Where $c$ is the light speed in vacuum, and $\Delta t_{0}$ is the time required for the light beam to pass the length of the stationary train for the stationary earth observer according to his clock. Now, if we substitute the value of $L$ in eq. (3) to eq. (1), we get

$$
\Delta t=\frac{c \Delta t_{0}}{\sqrt{c^{2}-v^{2}}}=\frac{\Delta t_{0}}{\sqrt{1-\frac{v^{2}}{c^{2}}}}
$$

Equation (4) indicates us that, for the stationary earth observer according to his earth clock, the time separation required for the light beam to pass the length of the moving train is greater than if the train is stationary by the factor of $\frac{1}{\sqrt{1-\frac{v^{2}}{c^{2}}}}$. Thus, eq. (4) indicates us also if the stationary earth observer registered by his clock a time separation for two events occurred inside the stationary train to be $\Delta t=\Delta t_{0}$, then, if this train moves with constant speed $v$, then the earth observer will register by his clock a time separation $\Delta t$ for the same two events to be occurred inside the moving train, where $\Delta t=\frac{\Delta t_{0}}{\sqrt{1-\frac{v^{2}}{c^{2}}}}$. Thus events are occurring inside the moving train in a slower rate than if the train was stationary for the stationary earth observer according to his earth clock according to eq. (4).

Now suppose both the earth observer and the observer on the moving train are agreed to perform this thought experiment. The observer on the moving train sent a ray of light along his moving train length, and both the earth observer and the observer on the moving train will measure the time required for the light beam to pass the length of the moving train, each one uses his clock. According to the MSRT $[25,26,27,43]$, both the earth observer and the observer on the moving train will be agreed at the moment of transmitting the ray of light from the back of the moving train and then they will be agreed at the moment of reaching the ray of light at the front of the moving train. We have seen previously, relative to the earth observer the direction of transmitting the light beam is independent on the direction of the velocity of the moving train. Also, both of them will be agreed at the measured length of the moving train to be $L$. Thus for the earth observer, the time separation of this two events (sending the light beam from back and then arriving the light to the front) according to his clock is given according to eq. (4). Where, the earth observer will measure a time separation for the light beam to pass the length of the moving train to be greater than if the train was stationary. Now for the observer on the moving train, since the motion of his clock inside the moving train is considered as events occurring inside the train, thus its motion will be slower when the train is moving than when it is at rest. And, since both the observer on the moving train and the stationary earth observer are agreed at the measured length of the moving train to be L, and also they are agreed at starting of sending the light beam from the back of the train and then agreed at the moment of reaching the light beam to the front of the moving train. Thus, by these conditions, when the stationary earth observer computed the time $\Delta t$ for the light beam to pass the length of the moving train $\mathrm{L}$, at this moment 
the observer on the moving train will measure the time separation $\Delta t^{\prime}$ according to his clock, where

And from eq. (4) we get $\Delta t^{\prime}=\sqrt{1-\frac{v^{2}}{c^{2}}} \Delta t$

$$
\Delta t^{\prime}=\Delta t_{0}
$$

Thus, eq. (5) indicates us that the observer on the moving train will measure a time separation for the light beam to pass his moving train length to be the same time separation if the train at rest. From that the measured speed of light inside the moving train for the observer on the moving train according to his clock locally is equal to the speed of light in vacuum, same as the stationary earth observer when he measures the speed of light on the earth surface; he will get it equals to the speed of light in vacuum. From that we get the main principle of the modified special relativity which illustrates the consistency of the speed of light locally.

* The speed of light is locally constant and equals to the speed of light in vacuum c for any inertial frame of reference.

From eq. (5), we can write eq. (4) as

$$
\Delta t=\frac{\Delta t^{\prime}}{\sqrt{1-\frac{v^{2}}{c^{2}}}}
$$

Equation (6) represents the equation of time dilation in Einstein's SRT.

\section{The LoRENTZ Transformation Equations and THE MSRT}

How can we understand the Lorentz transformation equations according to the MSRT in order to keep the laws of physics are the same for all inertial frames of reference?

We have seen in the previous section, when the light beam is passing through the moving train, then the time separation for passing the light beam the length of the moving train is independent on the direction of transmitting the light beam compared to the direction of the velocity of the moving train.

Both the stationary earth observer and the observer on the moving train are agreed at the length of the moving train to be $\mathrm{L}$, same as if the train is stationary. Also, both the stationary earth observer and the observer on the moving train are agreed at the moment of transmitting the light beam from the back of the moving train and also they will be agreed at reaching the light beam at the front of the train, and vice versa if the light beam was sent from the front to the end of the moving train.

From these postulates we derived eq. (6) which represents the equation of Einstein of the time dilation in the SRT.

Now suppose we have a tube full of water of length L. We have seen in optics, when a light beam is incident inside this tube, then the time separation for the light beam to pass the length of the tube is greater than if the tube is empty according to our lab clock. If the tube is empty and we measured the time separation $\Delta t_{0}$ by our clock for the light beam to pass the length of the tube, then when the tube is full of water, we shall measure the time separation $\Delta t$ where

$$
\Delta t=n \Delta t_{0}
$$

Where $\mathrm{n}$ is the refractive index of water. According to postulate $(*)$ and eq. (4), we get an equivalence when the light beam is passing through the moving train or passing through a medium of refractive index $n$. Suppose we have a meter stick of length $\Delta x_{0}$ in free space. If we put this meter stick inside the tube of water, we shall see the length of this meter stick is longer than in the free space, by the factor of $n$, the refractive index of water, where

$$
\Delta x=n \Delta x_{0}
$$

Where $\Delta x$ is the length of the meter stick inside the water for an observer in free space.

Thus from our equivalence principle, and from eq. (8), if we determined two points of length separation $\Delta x_{0}$ of the light path inside the train when it is stationary, then, when the train is moving with constant velocity $\mathrm{v}$, then, the measured length of the light path inside the moving train compared to the light path $\Delta x_{0}$ when the train is stationary for the stationary earth observer will be $\Delta x$ given according to eq. (8) as

$$
\Delta x=\frac{\Delta x_{0}}{\sqrt{1-\frac{v^{2}}{c^{2}}}}
$$

For the rider of the moving train the measured space and time separation inside his moving train for the light bath will be equal as it is stationary, where from eq. (5) we have $\Delta t^{\prime}=\Delta t_{0}$, and thus we get also

$$
\Delta x^{\prime}=\Delta x_{0}
$$

Thus from eq. (10), we can write eq. (9) as

$$
\Delta x=\frac{\Delta x^{\prime}}{\sqrt{1-\frac{v^{2}}{c^{2}}}}
$$

Equations (6) and (11) represent the measured space-time separation of the light bath inside the moving train comparing to the measured space-time of the light bath locally on the earth 
surface for the earth observer. For a free particle moving on the earth surface, the particle is defined by the space-time of $\Delta x$ and $\Delta t$ for the earth observer. But when this particle is incident inside the moving train, it will be defined locally by the spacetime of $\Delta x^{\prime}$ and $\Delta t^{\prime}$ of the stationary observer on the moving train locally. In this case $\Delta x$ is related to $\Delta x^{\prime}$ by (11), and $\Delta t$ is related to $\Delta t^{\prime}$ by (6). Now suppose a light beam is incident inside the moving train. According to the two points separated by a distance $\Delta x^{\prime}$ inside the moving train, for the rider of the moving train, the measured speed of light will be given as

$$
c^{\prime}=\frac{\Delta x^{\prime}}{\Delta t^{\prime}}=\frac{\Delta x_{0}}{\Delta t_{0}}=c
$$

$\Delta t^{\prime}$ is the time separation of the event for the rider according to his clock. Thus the rider will measure the light speed inside his moving train to be the light speed in vacuum.

For the stationary earth observer, within the same two points inside the moving train separated by a distance $\Delta x^{\prime}$ for the rider of the moving train, the measured speed of light will be given as

$$
c^{\prime \prime}=\frac{\Delta x}{\Delta t}=\frac{\sqrt{1-\frac{v^{2}}{c^{2}}} \Delta x^{\prime}}{\sqrt{1-\frac{v^{2}}{c^{2}}} \Delta t^{\prime}}=\frac{\Delta x_{0}}{\Delta t_{0}}=c
$$

Equation (13) indicates us; the measured speed of light inside the moving train for the earth observer will be equal to the speed of light in vacuum also! At the first time the reader will think eq. (13) is contradicted with eq. (2), but there is no contradiction. Since eq. (2) is predicting the light speed by measuring the time separation for the light beam to pass the length of the moving train according to the clock of the stationary earth observer. And since the length of the train is determined locally by the space on the earth surface and this length of the train is not changed if the train is moving or stationary. This is equivalent to the tube of length $L$ full of water. Suppose the length of the tube is 1 meter. Now if we have two meter sticks of length 1 meter. Now if we put one meter stick inside, along the water tube length and we put the other outside along the length of the tube. What shall we observe? We shall observe the meter stick inside the water will be appeared to be longer than the meter stick outside. And since the meter stick outside will give us the length of the tube locally. The meter stick inside will give us the length of the tube according to the space inside the tube. Thus, by using eqs. (7)\&(8) to determine the speed of light according to the spacetime inside the water tube, we get

$$
c^{\prime \prime}=\frac{\Delta x}{\Delta t}=\frac{n \Delta x_{0}}{n \Delta t_{0}}=c
$$

Equation (14) represents the measured speed of light inside the water tube according to the space-time coordinates inside the tube which is related to our coordinates according to eqs. (7)\&(8). Where, according to eq. (14), the measured speed of light is equal to the speed of light in vacuum. Equation (14) represents another interpretation why the light beam is taking longer time separation when it is passing though a medium of refractive index greater than 1 . According to the meter stick located outside along the length of the tube, the light speed will be decreased, and because of that it takes longer time separation according to our clocks. But according to the meter stick inside the medium, the light speed is the same light speed in vacuum, because the distance is longer inside the medium of refractive index greater than 1 according to eq. (8), so it takes longer time separation according to our clocks. Einstein in his special relativity adopted the second interpretation, the consistency of the speed of light and then the difference of measuring the time and space by the two observers who are moving in a relative velocity. But, what we have discovered in our MSRT that the two interpretations are equivalent to each others as we shall see in the following.

In order to understand how my theory works, let's start studying the thought experiment which was adopted by Einstein's SRT which illustrating his interpretation of the Lorentz transformation equations and the relative simultaneity. Suppose both the earth observer and the observer of the moving train will perform this thought experiment. As in fig. (1), at pylon A the train started to move with constant speed $\mathrm{v}$, and at this moment the observer stationary on the moving train sent a ray of light from back to front the train, and also at this time the observer on the ground sent a ray of light from pylon A to pylon B. The two rays of light are sent along the direction of the velocity of the train.

Relative to the observer stationary on the moving train, the distance between the back and the front is fixed, where the length of his moving train is the same length as if the train is stationary. For the observer on the ground, while he sees the ray of light moves toward the front of the moving train, he will see also at the same time the front of the moving train is going far from the light beam, that is because the train moves with constant speed $\mathrm{v}$ at the same direction of the light beam. In this case and according to the concept of the classical relativity, the observer stationary on the train will see the light beam arrives the front of his moving train before the observer stationary on the ground. Let's propose this conditionaccording to the classical relativity- if at the moment that the observer stationary on the moving train sees the light beam reaches to the front of the train, at this moment the train arrives to the second pylon B on the ground. According to the concept of the objective existence of the phenomenon in the classical physics, both the observer on the ground and the observer stationary on the moving train will see the train arrives pylon $\mathrm{B}$, but according to the classical relativity, the observer stationary on the moving train will see the light beam arrives to the front of his train and also at this time the front of his train arrives to pylon B. But at this moment for the observer stationary on the ground, he sees the train's front 
arrives to pylon $\mathrm{B}$, but the light beam is still approaching to pylon B.

The negative result of the Michelson-Morely experiment [38] illustrated the speed of the light beam will not be affected by the relative velocity and thus both the observer stationary on the ground and the observer stationary on the moving train must see the light beam arrives the front of the moving train and the front must arrive to pylon B at the same moment according to the concepts of the classical physics at that time.

And thus in order to solve the negative result of the Michelson-Morely experiment FitzGerald [38,39] Proposed the concept of the length contraction in the direction of the velocity, where according to this concept the length of the moving train must be contracted along the direction of the moving train relative to the observer on the ground, and thus according to this concept both the observer on the ground and the observer on the moving train will see the light beam arrives the front of the train, and also the front of the moving train arrives pylon B. After that, Lorentz [40, 41, 42] proposed his transformation in order to keep on the constancy of the speed of light and then the invariance of Maxwell's equations and the laws of physics. Einstein interpreted the Lorentz transformation equations according to the concept of the relative velocity and the simultaneity by his special relativity SRT. According to the SRT of Einstein, both the observer on ground and the observer on the moving train see the light beam arrives to the front of the moving train and the front of the train arrives to pylon (B) as well, but in a different time separation and space separation. Where according to the reciprocity principle of the SRT of Einstein and under the postulate of the constancy of the speed of light, he defined the Lorentz contraction in the direction of the velocity, where the measured length of the moving train for the observer on the ground is $\mathrm{L}^{\prime}$ where

$L^{\prime}=\sqrt{1-\frac{v^{2}}{c^{2}}} L$, where $L$ is the proper length of the moving

train for the observer stationary on the moving train, where $\mathrm{L}$ is equal to the length of the train if it was stationary. Thus from that and according to the SRT of Einstein's interpretation of the Lorentz contraction, both the observer on the ground and the observer on the moving train will agree that the light beam arrives to the front of the train and also the front of the train arrives to pylon $B$. Now, relative to the light beam that was sent from pylon $\mathrm{A}$, according to the classical relativity, for the observer stationary on the moving train, pylon $\mathrm{A}$ is moving with velocity $-\mathrm{v}$ relative to him, and thus the light beam is going far from the front of his train. Thus, at the moment that the stationary observer on the train sees the front of his train arrives at pylon $B$, he will see the light beam that was sent from pylon A is not arrived to pylon B according to classical relativity. According to classical relativity the observer stationary on the moving train will see the light beam is still approaching to pylon B, while at this moment, the observer on the ground sees the light beam which was sent from pylon $\mathrm{A}$ is arrived to pylon $\mathrm{B}$, and he agrees also with the observer stationary on the moving train that the front of the train arrives to pylon B. According to Einstein's SRT interpretation of the Lorentz contraction, both the observer on the ground and the observer stationary on the moving train will agree that the light beam which was sent from $A$ reaches to pylon B but they will be different in the time separation of the event and the space separation between the two pylons, where the observer stationary on the moving train will measure the distance between the two pylons to be contracted.

According to Einstein's interpretation to the Lorentz transformation in his SRT, it is impossible measuring a particle or electromagnetic waves to move faster than light. Where, that leads to violation the Lorentz invariance and causality. In this paper I'll introduce a new interpretation to the Lorentz transformation depending on a new understanding to the Lorentz transformation equations. And this interpretation is leading to the possibility of measuring faster than light without violation of the Lorentz invariant or causality and at the same time keeping on the constancy of the speed of light to be $c$ the speed of light in vacuum locally.

In order to understand my new interpretation to the Lorentz transformation in my MSRT, let's study the previous thought experiment of Einstein which illustrated a light-like interval. Suppose again both the earth observer and the observer of the moving train will perform this thought experiment. As in fig. (1), at pylon A the train started at rest to move with constant speed $\mathrm{v}$, and at this moment the observer stationary on the moving train sent a ray of light from back to front the train, and also at this time the observer on the ground sent a ray of light from pylon A to pylon B. The two rays of light are sent along the direction of the velocity of the moving train

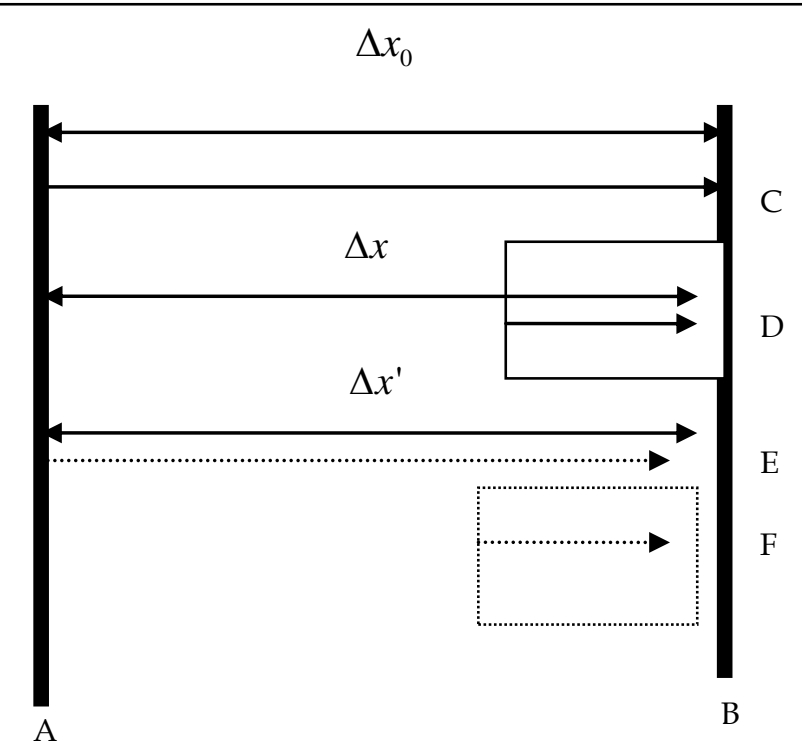

Fig. (1): at the moment that the train started at rest to move with speed $v$ from pylon $A$ to $B$, a ray of light was sent from pylon $A$ to pylon $B$ and at the same time a ray of light was sent inside the moving train from back to front. The distance between the two pylon is $\Delta x_{0}$ 
According to my new Lorentz transformation equations, if the observer on the ground sees the front of the moving train arrives to pylon $\mathrm{B}$ and passed the distance $\Delta x_{0}$ between the two pylons, at this moment he sees the light beam which sent from back to front does not arrive to the front, where if the length of the moving train is $L$, then the light beam is at a distance $L^{\prime}$ from the back of the moving train -at point D in fig. (1)- Where

$$
L^{\prime}=\sqrt{1-\frac{v^{2}}{c^{2}}} L
$$

$$
\Delta x=\sqrt{1-\frac{v^{2}}{c^{2}}} \Delta x_{0}
$$

While, at this moment the observer on the ground sees the ray of light that was sent from pylon A arrives to pylon B at point $\mathrm{C}$ at a time separation $\Delta t_{0}$ according to his clock on the ground, where

$$
\Delta t_{0}=\frac{\Delta x_{0}}{c}
$$

Where $c$ is the speed of light in vacuum. At this moment for the observer on the moving train during the motion, the front of the moving train does not arrive pylon B, but it is still approaching to it, where he sees the front of his moving train is at a distance $\Delta x^{\prime}$ from pylon $\mathrm{A}$, The dotted line of the moving train in fig. (1) illustrates the location of the front of the moving train for the observer on the moving train at the moment that the observer on the ground sees the front of the moving train arrives pylon $B$, where in this case we have

$$
\Delta x^{\prime}=\sqrt{1-\frac{v^{2}}{c^{2}}} \Delta x_{0}
$$

And at this moment for the observer on the moving train, his clock on the moving train registers the time separation $\Delta t^{\prime}$ where

$$
\Delta t^{\prime}=\sqrt{1-\frac{v^{2}}{c^{2}}} \Delta t_{0}
$$

Also at this moment the observer on the moving train sees the light beam which was sent from back does not reach the front, where it is still at a distance

$$
L^{\prime}=\sqrt{1-\frac{v^{2}}{c^{2}}} L \text { from the back (point } F \text { in fig. (1)), and he }
$$

sees at this moment the light beam which sent from pylon $A$ is moving with the same time with the light beam which sent from the back (point E in fig (1)).

Since the light beam that was sent from back to front is sent in the same direction of the velocity of the moving train, thus for the observer on the ground and from fig. (1), we have

$$
\Delta x=\Delta x_{0}-v \Delta t_{0}
$$

Since the light speed on the moving train is $\mathrm{c}$ the speed of light
And

$$
\begin{gathered}
\Delta x=\frac{\Delta x^{\prime}}{\sqrt{1-\frac{v^{2}}{c^{2}}}}-\frac{v \Delta t^{\prime}}{\sqrt{1-\frac{v^{2}}{c^{2}}}} \\
\Delta t=\Delta t_{0}-\frac{v \Delta x_{0}}{c^{2}}
\end{gathered}
$$

Thus from eqs. (16)\&(17) we get

in vacuum locally for the observer on the moving train, thus from eqs. (16) \&(17) we get

$$
\Delta t=\frac{\Delta t^{\prime}}{\sqrt{1-\frac{v^{2}}{c^{2}}}}-\frac{v \Delta x^{\prime}}{c^{2} \sqrt{1-\frac{v^{2}}{c^{2}}}}
$$

In my new interpretation to the Lorentz transformation in the case of $y$ and $z$ axis we have

$$
\begin{gathered}
\Delta y=\frac{\Delta y^{\prime}}{\sqrt{1-\frac{v^{2}}{c^{2}}}} \\
\Delta z=\frac{\Delta z^{\prime}}{\sqrt{1-\frac{v^{2}}{c^{2}}}}
\end{gathered}
$$

The change in $\mathrm{y}$ and $\mathrm{z}$ coordinates in my transformation leads to illustrate the negative result in the Michelson-Morley experiment.

This transformation which we adopted illustrated that the Lorentz transformation is vacuum energy dependent. We have seen that both the observer on the moving train and the observer on the ground will be agreed at the measured length of the moving train to be $L$ same as if the train is stationary, and at the same time they will be agreed at the length between the two pylons to be $\Delta x_{0}$ during the motion.

For the observer on the moving train, when he sees the front of his moving train arrives pylon B, he sees also at this moment the light beam which sent from back arrives the front of his moving train and the passed distance at this moment for him is $\Delta x^{\prime}=\Delta x_{0}$ which leads to eq. (10), in a time separation

$$
\Delta t^{\prime}=\Delta t_{0}
$$

Which leads to eq. (5), according to his clock on the moving train. And at the same time he sees the light beam which sent from pylon A arrives pylon B, where the light beam which sent from pylon A moves at the same time with the light beam which sent from back to front. At this moment- during the motion- the observer on the ground sees the moving train passed pylon $B$ and it is at distance

$$
\Delta x=\frac{\Delta x_{0}}{\sqrt{1-\frac{v^{2}}{c^{2}}}}
$$


from pylon A, which leads to eq. (9), and his clock on the ground registered a time separation

$$
\Delta t=\frac{\Delta t_{0}}{\sqrt{1-\frac{v^{2}}{c^{2}}}}
$$

which leads to eq. (4), where at this time the observer on the ground sees the light which sent from back arrives the front of the moving train. This is agreed and interpreting with we proposed in section 2, where at this moment both the observer on the ground and the observer on the moving agree that the light beam which sent from the back arrives the front of the moving train.

According to our interpretation to the Lorentz transformation, it is impossible during the motion that both observer on the ground and the observer on the moving train agree that the train arrived pylon B at the same time. This is the core of the Heisenberg uncertainty principle when we deal with the four vectors in my new interpretation to the Lorentz transformation.

Now let's propose another thought experiment. Suppose the train moves from pylon A to pylon B and the ray of light is sent from pylon $B$ to pylon $A$ in the opposite direction of the velocity, and at the same time a ray of light is sent inside the moving train from front to back as in fig. (2) in the opposite direction of the velocity. According to my MSRT interpretation to the Lorentz transformation, and according to fig. (2), when the train front arrives to pylon B and the moving train passed the distance $\Delta x_{0}$ between the two pylons relative to the observer stationary on the ground, at this moment for the observer on the moving train, the train is at distance $\Delta x^{\prime}$ from pylon A where

$$
\Delta x^{\prime}=\sqrt{1-\frac{v^{2}}{c^{2}}} \Delta x_{0}
$$

as in eq. (16), in a time separation $\Delta t^{\prime}$ according to his clock where

$$
\Delta t^{\prime}=\sqrt{1-\frac{v^{2}}{c^{2}}} \Delta t_{0}
$$

as in eq. (17).

Thus, at this moment the observer on the ground sees the light beam which sent from the front does not arrive the back of the moving train. If the length of the moving train is $L$, where $L$ is the same length if the train is stationary for the observer on the ground, then the light beam is at a distance $L^{\prime}$ from the front (point D in fig. (2)), where

$$
L^{\prime}=\sqrt{1-\frac{v^{2}}{c^{2}}} L
$$

And from fig. (2), we get also

$$
\Delta x=\sqrt{1-\frac{v^{2}}{c^{2}}} \Delta x_{0}
$$

At this time also, the observer on the ground sees the light beam which sent from pylon $B$ arrives pylon $A$ (point $C$ in fig. (2)). Now, since the light beam is sent in the apposite direction of the velocity of the moving train inside the moving train, then we have for the observer on the ground

$$
\begin{aligned}
& \Delta x=\Delta x_{0}+v \Delta t_{0} \\
& \Delta t=\Delta t_{0}+\frac{v \Delta x_{0}}{c^{2}}
\end{aligned}
$$

And thus from eqs. (16) \&(17) we get

And

$$
\begin{gathered}
\Delta x=\frac{\Delta x^{\prime}}{\sqrt{1-\frac{v^{2}}{c^{2}}}}+\frac{v \Delta t^{\prime}}{\sqrt{1-\frac{v^{2}}{c^{2}}}} \\
\Delta t=\frac{\Delta t^{\prime}}{\sqrt{1-\frac{v^{2}}{c^{2}}}}+\frac{v \Delta x^{\prime}}{c^{2} \sqrt{1-\frac{v^{2}}{c^{2}}}} \\
\Delta y=\frac{\Delta y^{\prime}}{\sqrt{1-\frac{v^{2}}{c^{2}}}} \\
\Delta z=\frac{\sqrt{\Delta z^{\prime}}}{\sqrt{1-\frac{v^{2}}{c^{2}}}}
\end{gathered}
$$

At this moment for the observer on the moving train, the light beam which sent from the front is at distance L' from the front, where it does not arrive the back yet, where $L^{\prime}=\sqrt{1-\frac{v^{2}}{c^{2}}} L$ (point $\mathrm{F}$ in fig. (2)), and he sees at this time also the light beam which sent from pylon B is moving with the same time with the light beam which sent from the front, where when the observer on the moving train sees the front of the his train arrives pylon $\mathrm{B}$, he sees also at the same time the light beam which sent from the front arrives the back, and also at the same time he sees the light beam which sent from pylon B arrives pylon $\mathrm{A}$. In this case for the observer on the moving train

$$
\Delta x^{\prime}=\Delta x_{0}
$$

Which leads to eq. (10), and $\Delta t^{\prime}=\Delta t_{0}$ according to his clock on the moving train, which leads to eq. (5).

At this time for the observer on the ground the front of the moving train passed pylon $\mathrm{B}$, and it is at a

distance $\Delta x$ from pylon A, where $\Delta x=\frac{\Delta x_{0}}{\sqrt{1-\frac{v^{2}}{c^{2}}}}$, which 
leads to eq. (9). in time separation $\Delta t$ according to his earth clock, where

$$
\Delta t=\frac{\Delta t_{0}}{\sqrt{1-\frac{v^{2}}{c^{2}}}}
$$

which leads to eq. (4), and at this time the observer on the ground sees the light beam which sent from the front arrives the back of the moving train. This is agreed and interpreting with what we discussed in section 2. That interprets our proposition, when we proposed in section 2 , if the light beam is sent inside the moving train in the direction of the velocity or in the opposite direction of the velocity, then both the stationary observer on the ground and the observer on the moving train are agreed that light beam is sent from the first edge of the moving train and then they will be agreed at arriving the light to the second edge but in a different space and time separation during the motion.

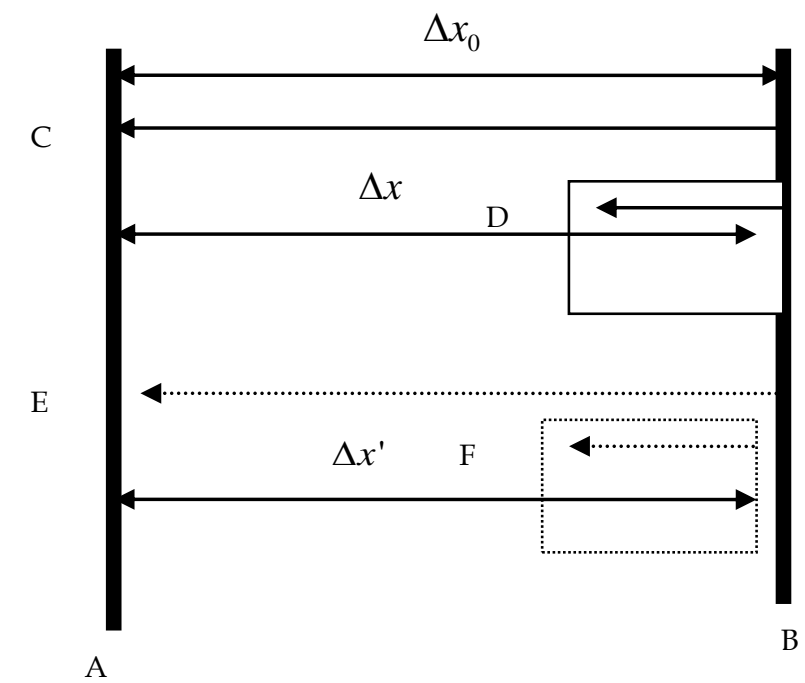

Fig. (2): The train started at rest to move from pylon A to Pylon $B$. At this moment a light beam was sent from front to back in the opposite direction of the velocity. Also a light beam was sent from pylon $B$ to pylon $A$.

In my new interpretation to the Lorentz transformation we keep on the consistency of the speed of light locally to be the speed of light in vacuum. In my proposition, I adopted the principle of quantum theory (Copenhagen school) that the observer has the main formation of the phenomenon. And I refuse in my proposition the principle of the objective existence of the phenomenon that is existed in Einstein's SRT and then his interpretation to Lorentz transformation equations. In my proposition, both the observer on the ground and the rider of the moving train will agree at the measured length of the moving train to be $L$ same if the train was stationary and then at the measured distance between the two pylons $\mathrm{A} \& \mathrm{~B}$, where each observer creates his own picture about the location of the moving train and the time separation. According to my proposition in my modified relativity I predicted also the observer on the moving train will see the clock of the observer on the ground is moving in a similar rate of his clock motion on the moving train, that means the observer on the moving train sees the events on the ground in his present which are considered as past relative to the observer on the ground as we shall see in the following.

According to my new interpretation to the Lorentz transformation, we have seen for the observer on the ground, according to his clock, when the light beam is sent from pylon A to pylon B, it takes less time separation than when the light beam is sent from back of the moving train to the front in the same direction of the velocity. Also when the light beam is sent from pylon $B$ to pylon $A$ it takes less time separation than when the light beam is sent from front to back inside the moving train in the opposite direction of the velocity of the moving train. In this case we have seen for the observer on the ground the time separation for the light beam to pass the length of the moving train from back to front in the same direction of the velocity is the same time separation for the light beam to pass the length of the moving train from front to back in the opposite direction of the velocity of the moving train according to the clock of the observer on the ground. Thus according to my new interpretation to the Lorentz transformation we have get if the light beam is sent inside the moving train in any direction in the $\mathrm{x}, \mathrm{y}$, or $\mathrm{z}$, then the measured speed of light $c^{\prime}$ for the observer on the ground according to his clock is independent on the direction of the velocity of the moving, and thus we get

$$
c^{\prime}=\sqrt{1-\frac{v^{2}}{c^{2}}} c=\sqrt{c^{2}-v^{2}}
$$

This leads to eq. (2) according to my new interpretation to the Lorentz transformation equations according to my modified relativity.

Since my new interpretation to the Lorentz transformation leads to the wave-particle duality, thus if the observer on the moving train sent a particle of velocity $v_{p}$ locally which is equivalent to the relativistic kinetic energy $E_{k}$, in this case the measured speed of the particle for the observer on the ground when the particle passed all the length of the moving train inside the moving train according to his clock is $v_{p}^{\prime}$

$$
v_{p}^{\prime}=\sqrt{1-\frac{v^{2}}{c^{2}}} v_{p}
$$

Where according to the eq. (21) for the observer on the ground the measured velocity of the moving particle inside the moving train is independent on the direction of the velocity of the train same as in the case of the light beam, and it is less than the equivalent velocity of the kinetic energy of the particle and when the particle exits the moving train, that causes a red shift or blue shift measured by the observer on the ground. In this case the measured Doppler Effect will be slightly different from the computed by Einstein SRT according to eq. (21). 
The dependence of my transformation on the vacuum energy can be illustrated from the measured relativistic volume of the moving train, where from my Lorentz transformation the measured relativistic volume of the moving train for the observer on the ground is given as

Thus

$$
V=\Delta x \Delta y \Delta z=\frac{\Delta x^{\prime} \Delta y^{\prime} \Delta z^{\prime}}{\left(1-\frac{v^{2}}{c^{2}}\right)^{3 / 2}} \pm \frac{\Delta y^{\prime} \Delta z^{\prime} v \Delta t^{\prime}}{\left(1-\frac{v^{2}}{c^{2}}\right)^{3 / 2}}
$$

$$
V=\frac{V^{\prime}}{\left(1-\frac{v^{2}}{c^{2}}\right)^{3 / 2}} \pm \frac{A^{\prime} v \Delta t^{\prime}}{\left(1-\frac{v^{2}}{c^{2}}\right)^{3 / 2}}
$$

Here $\mathrm{V}$ is the relativistic volume of the moving train for the observer on the ground, $\mathrm{V}^{\prime}$ is the measured volume locally for the rider of the moving train, and $\mathrm{A}^{\prime}$ is the area of the front or the back of the moving train Locally for the rider. Since both of the observer on the ground and the rider of the moving train are agreed at the lengths of the moving train in $x, y$ and $z$ direction locally, thus, the equation above illustrating the increase of the vacuum energy of the moving train for the observer on the ground, and that leads to the Lorentz factor is equivalent to the refractive in optics or the potential in Schrödinger equation in quantum.

My interpretation of the Lorentz transformation illustrates what is the wave-function of Heisenberg who defined it as "it is a mixture between two things, the first is the reality and the second our knowledge to this reality" [1]. My paper "The Comalogical Theory" [29, 30] illustrates the philosophical aspects of my modified relativity and how it is related to the Copenhagen school in quantum theory. What I proposed in my Lorentz transformation illustrates what is the interference and diffraction in optics which led to discovering the wave mechanics in quantum theory.

In the literature of relativity, space-time coordinates and the energy/momentum of a particle are often expressed in fourvector form. They are defined so that the length of a fourvector is invariant under a coordinate transformation. This invariance is associated with physical ideas. The invariance of the space-time four-vector is associated with the fact that the speed of light is a constant. The invariance of the energymomentum four-vector is associated with the fact that the rest mass of a particle is invariant under coordinate transformations. In our previous thought experiments we proposed a null vector or lightlike. In our previous examples, the null vector for the observer stationary on the moving train according to his coordinates system is

$$
c^{2} \Delta t^{\prime 2}-\Delta x^{\prime 2}-\Delta y^{\prime 2}-\Delta z^{\prime 2}=0
$$

and then by my new Lorentz transformation equations we have also relative to the observer on the ground

$$
c \Delta t^{2}-\Delta x^{2}-\Delta y^{2}-\Delta z^{2}=0
$$

We have seen previously, the Lorentz transformation is applied during the motion, and as we have seen in our previous examples, when the observer on the ground sees the front of the moving train arrives to pylon B, at this moment it is impossible that the observer on the moving train sees the front of the moving train arrives pylon $B$, both of them are not agreed that the front of the moving train arrives to pylon $\mathrm{B}$, and this is the main difference between my MSRT and the SRT of Einstein. According to my MSRT when the observer on the moving train looks at the clock stationary on the ground, he will see the clock on the ground is moving in a similar rate of his clock motion, and since his clock is considered stationary on the moving train, then he will confirm that the clock on ground is stationary also same as his clock on the moving train. From that also he will agree with the observer on the ground on the measured rest mass of the clock. When the clock on the ground moves with speed $\mathrm{v}$ on the ground, in this case both the observer on the ground and the observer on the moving train are agreed at measured speed of the moving clock and then the relative measured mass, and the kinetic energy and the momentum, and the clock is moving slower than their clocks and they will agree at the slowing rate. Furthermore according to my MSRT, I could rescue the special relativity from the Twin paradox, Ehrenfest paradox, Ladder paradox and Bell's spaceship paradox as we have seen previously.

From that we can define in a simple meaning the Heisenberg uncertainty principle according to my interpretation to the Lorentz transformation equations and how that is related to the vacuum energy. Where, if the train at rest started to move from pylon $A$ to pylon $B$ in constant velocity $v$, then it is impossible during the motion that the observer on the moving train and the observer on the ground agree that the moving train arrives to pylon $B$ simultaneously, which refusing the objective existence of the phenomenon that adopted by Einstein SRT. Because of that Einstein said at the end of his life; I hate the Heisenberg uncertainty principle. Einstein knew the consequences of Heisenberg uncertainty principle on his SRT.

\section{The Length Contraction According to MSRT}

To understand the concept of the length contraction according to the MSRT [25, 26, 27, 43], let's assume Sally is driving a train with constant velocity $0.87 \mathrm{c}$ between the two pylons $\mathrm{A} \& \mathrm{~B}$, and the distance between the two pylons is 100 $\mathrm{m}$. let's assume also at the moment of reaching the train at pylon B, Sara who was stationary on the earth could stop the train instantaneously by a remote control. In this case we neglect the deceleration because this case is equivalent to some cases in quantum as we shall see in following sections. Thus, in this case we consider the velocity of the train is changed from $0.87 \mathrm{c}$ to zero in a zero time separation at the moment of reaching to Pylon $B$. Thus, by this condition we have

$$
\begin{gathered}
v=0 \text { at } L=0 \\
v=0.87 c \text { at } 0<\mathrm{L} \leq 100 \mathrm{~m} \\
v=0 \text { at } L=100 \mathrm{~m}
\end{gathered}
$$


The concept of the length contraction which is adopted by the MSRT [27] is agreed with the concepts, principles and laws of quantum theory (Copenhagen School) .

Subsequently, according to MSRT [27] during the motion, when Sara sees the train reached to pylon B, at this moment Sally will not see the train reached at the second pylon $B$, it is still in the middle of her trip at $50 \mathrm{~m}$ from pylon $\mathrm{A}$, and thus it is still approaching to the second pylon B. Subsequently, according to this interpretation, when Sara sees the moving train at a distance $\Delta x$, at this moment Sally will see her moving train is at the distance $\Delta x^{\prime}=\sqrt{1-\frac{v^{2}}{c^{2}} \Delta x}$. This interpretation is agreed with the concept of Heisenberg to the wave function, where the observer has the main formation of the phenomenon. And by this interpretation Sally and Sara create their own pictures about the location of the moving train. Now, for Sara, the measured velocity of the moving train is given as $v=\frac{\Delta x}{\Delta t}=0.87 c$ which is equal to the equivalent velocity of the kinetic energy owned by the moving train. For Sally (who is the driver of the train) there are two states that the train existed instantaneously, the first one is the state of motion, and the measured velocity of the train at this state for Sally is given as

$$
v^{\prime}=\frac{\Delta x^{\prime}}{\Delta t^{\prime}}=\frac{\sqrt{1-\frac{v^{2}}{c^{2}} \Delta x}}{\sqrt{1-\frac{v^{2}}{c^{2}} \Delta t}}=v=0.87 c
$$

And this measured velocity is equal to the measured velocity equivalent to the kinetic energy owned by the moving train. The other state is the state of stationary, and the predicted velocity of the train for Sally at this state is given as

$v^{\prime}=\frac{\Delta L^{\prime}}{\Delta t^{\prime}}=\frac{\Delta L}{\sqrt{1-\frac{v^{2}}{c^{2}} \Delta t}}=\frac{v}{\sqrt{1-\frac{v^{2}}{c^{2}}}}=\frac{0.87 c}{\sqrt{1-(0.87)^{2}}}=1.74 c$

Those two states of the train are separated by a distance equals to 50m, where Sally will think her train passed this distance in a zero time separation as seen in fig. 3 , and then Sally will think the distance of $100 \mathrm{~m}$ was passed by her train with velocity equals to $1.74 \mathrm{c}$ which is greater than the speed of light in vacuum. This measured velocity is not real, as we have seen the train hasn't moved with speed greater than the speed of light in vacuum locally for Sara, but because of the time dilation, and as we have seen in eqs. (4)\&(6), events are occurring in the frame of the moving train in a slower rate than on the earth surface, and then the clock of the moving train will compute a time separation of the event less than the earth clock. The difference of time between what is computed by the train clock of Sally at the state of stationary, and what is computed by the earth clock of Sara for the train to pass the distance $100 \mathrm{~m}$, we find this difference is negative, and this difference led Sally to think her train passed the distance $100 \mathrm{~m}$ between the two pylons with speed greater than the speed of light in vacuum. From fig. 3, Sally would confirm that the distance between the interval $50<x^{\prime}<100 \mathrm{~m}$ was not passed by her train. Her train was transformed from $50 \mathrm{~m}$ to $100 \mathrm{~m}$ in a zero time separation. For Sally time is contracted!

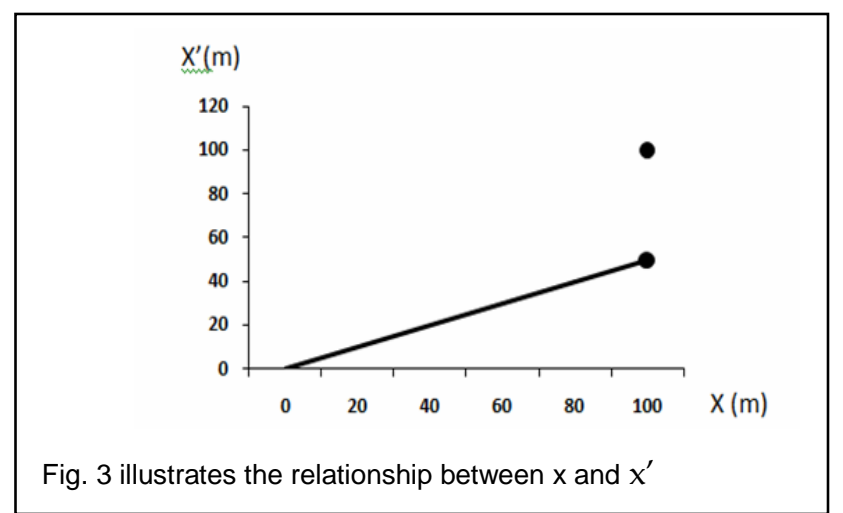

There is another consequence that produced by adopting this interpretation of the length contraction by MSRT [25, 26, 27, 43]. It is; how does Sally see the motion of Sara's earth clock comparing to her clock during the motion. According to MSRT [25, 26, 27, 43], Sally will see the motion of the earth clock of Sara is moving similar to her moving train clock, and by adopting this principle let's study the following thought experiment.

Suppose Sally during the motion of her train is looking at the stationary earth clock of Sara by applying this condition

$$
\begin{gathered}
v=0 \text { at } \Delta t_{\text {Sara }}=0 \\
v=0.87 c \text { at } 0<\Delta t_{\text {Sara }} \leq 4 \text { years. } \\
v=0 \text { at } \Delta t_{\text {Sara }}>4 \text { years }
\end{gathered}
$$

Where $\Delta t_{\text {Sara }}$ is the reading of Sara from her clock. We can draw $\Delta t_{\text {Sara }}$ versus $\Delta t_{\text {Sally }}$ as in fig. 4 , where $\Delta t_{\text {Sally }}$ is the reading of Sally from the clock of Sara. From fig. 4, we find two straight lines; the first one is for $0<\Delta t_{\text {Sara }} \leq 4$ yearsand its slope is equal to 0.5 . The second line is for $\Delta t_{\text {Sara }}>4$ years, and its slope is equal to 1 . We find from fig.4, the years between $2<\Delta t_{\text {Sara }} \leq 4$ years would not be determined by Sally, where her train was stopped at $\Delta t_{\text {Sara }}>4$ years, and thus she would find that Sara is living the years at $\Delta t_{\text {Sara }}>4$ years, while her last reading was equal to 2 years. That means the events were lived by Sara between $2<\Delta t_{\text {Sara }} \leq 4$ years were not be received by Sally during her motion. 


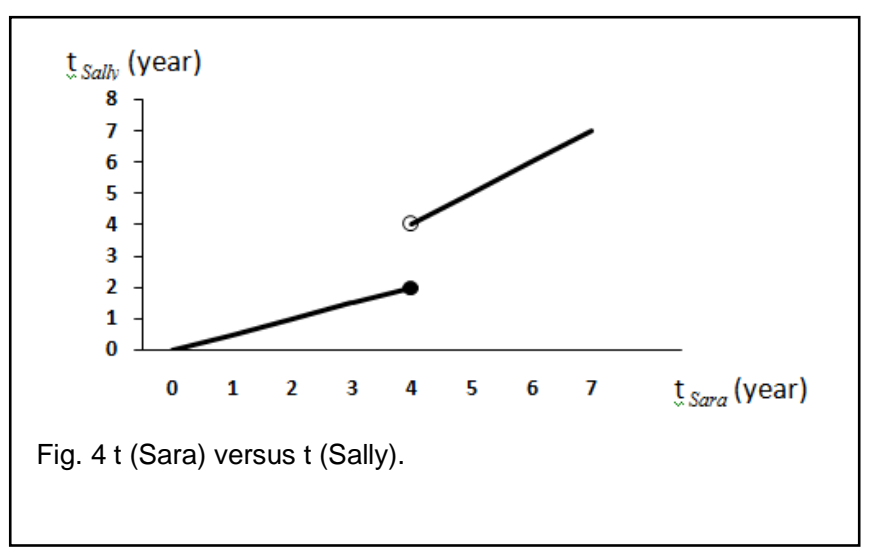

From the fig. 4 we get, the observer is the main participant in formulation of the phenomenon, where each one creates his own clock picture during the motion although they used the same clock. That is in contrast with the objective existence of the phenomenon.

\section{The Quantization OF General Relativity}

\section{INTRODUCTION}

We have seen previously a new interpretation for the Lorentz transformation equations, which leading to the Lorentz transformation is vacuum energy dependent instead of the relative velocity in SRT, and the Lorentz factor is equivalent to the refractive index in optics. In the previous sections, we have proposed an inertial frame of reference. That means the frame is moving with constant velocity or stationary. Now if the frame is accelerated or decelerated, then according to our new transformation the vacuum energy will be changed, and that is equivalent to the fluctuations of the vacuum as in quantum theory. This change in the vacuum energy of the accelerated or decelerated frame is controlled by quantum laws, and the Lorentz factor equivalent to the difference infinities as in the case of Casimir effect as we shall see in the following sections in my equivalence principle. This difference of vacuum energy maybe negative which leading to faster than light in the case of refractive index less than 1 , or positive which leading to time dilation, and the decreasing the speed of light as in optics in the case of refractive index greater than 1.

\subsection{The Relativistic Quantized Force}

Newton's Second Law of motion defined that the force acts on a body equals to the product of the rest mass of the body with its acceleration [9], and the acceleration is given as the second derivative for a distance with respect to time. When Einstein reached to his special theory of relativity in 1905 , he reached to the measuring of the relativistic mass, which indicates that the mass of the body increased with increasing the speed of the body $[4,7,15]$. Einstein depends on his relativistic equations derivation on the classical physical conceptions, which depend on the determinism, causality and continuity [11,12], and also depend on the possibility of measuring the velocity and the position of the particle simultaneously $[4,7,11,12,15]$. And depending on these concepts Einstein interpreted the Lorentz transformation equations in his SRT in the case of inertial frames of reference. But Heisenberg uncertainty principle assures the impossibility of measuring the velocity and the position simultaneously according to quantum theory. And then quantum theory proved the uncausality, indeterminism and the discontinuity in the micro world. That was violating the concepts and principles of the Einstein's interpretation to the Lorentz interpretation in the SRT $[1,2,5,12,14]$. We conclude from that according to quantum theory and the Heisenberg uncertainty principle, for measuring the velocity or the momentum for any body, we should know the energy equivalent to the relativistic kinetic energy held by this body, or the equivalent frequency for this energy. Since, according to the uncertainty principle, it is possible measuring the momentum and the energy simultaneously, therefore it is possible expressing the momentum in terms of the equivalent frequency of this energy to this body $[1,2,5,12,14]$. The force that affected on a body is given through the momentum first derivative with respect to time. Subsequently, we can express the momentum of the body in terms of frequency and time, and then we can get the applied force as the first derivative of the momentum with respect to time. Then we get the applied force in terms of equivalent frequency to the energy which coring by the body.

The cycle number of a standing electromagnetic wave in terms of time [8] is given by the relation

$$
n=v t
$$

Where $n$ is the cycle number at a time $t$, and $v$ is the wave frequency[8]. The time $t$ in eq. (5.1.1) is defined by the relation

$$
t=N\left(\frac{1}{2 v}\right)
$$

where

$$
N=1,2,3, \ldots, \frac{2 v}{v_{u}}
$$

Where $v_{u}$ is the frequency unit, where $v_{u}=\frac{1}{t_{u}}$, and $t_{u}$ is the time unit. From the eqs. (5.1.1) and (5.1.2) we get

$$
n=\frac{N}{2}
$$

We find from eq. (5.1.3) that $n$ takes the values $\frac{1}{2}, 1, \frac{3}{2}, 2, \ldots ., \frac{v}{v_{u}}$. Since the frequency is defined as the number in the unit of time, subsequently, when $t=t_{u}$ in eq. (5.1.2) we get

$$
N=2 v t_{u}
$$

and from this we get

$$
n=\frac{v}{v_{u}}
$$

The energy of the electromagnetic wave is defined by the relation 


$$
E=h v
$$

Where $E$ is the energy and $h$ is Plank's constant $[5,6]$, and from eqs. (5.1.4) and (5.1.5) we get

$$
E=\frac{N}{2} h v_{u}=n h v_{u}
$$

And by putting $H=h v_{u}$, we get

$$
E=N \frac{H}{2}
$$

And also

$$
E=n H
$$

Equation (5.1.7) indicates that, the energy of the standing electromagnetic wave takes integral value of $\frac{H}{2}$, and from that we can get the minimum energy $E_{\min }$ for the stating electromagnetic wave, and that is when $N=1$, where we get

$$
E_{\text {min }}=\frac{H}{2}
$$

when the energy value equals to $H$, it is called $H$-energy, where $H=6.626 \times 10^{-34}$ joule, and the equivalent mass to the $H$-energy is given by

$$
m_{H}=\frac{H}{c^{2}}
$$

Where $m_{H}$ is the equivalent mass for $H$-energy, and the equivalent mass is called $H$-particle. The relativistic kinetic energy $E_{k}[15]$ for a body moving with constant velocity $\mathrm{V}$ is given by

$$
E_{k}=\frac{m_{0} c^{2}}{\sqrt{1-\frac{V^{2}}{c^{2}}}}-m_{0} c^{2}
$$

Here we used V (Capital letter) to indicates for velocity, and $v$ to indicates it to frequency.

And by substituting the value $E_{k}=n H$ as in eq. (5.1.8), and $m_{0} c^{2}=n_{0} H$ in the last equation we get

$$
n H=\frac{n_{0} H}{\sqrt{1-\frac{V^{2}}{c^{2}}}}-n_{0} H
$$

And from (5.1.10), we get

$$
\frac{n_{0}}{\sqrt{1-\frac{V^{2}}{c^{2}}}}=n_{0}+n
$$

multiplying both sides of equation (5.1.11) by $m_{H}$, we get

$$
\frac{n_{0} m_{H}}{\sqrt{1-\frac{V^{2}}{c^{2}}}}=m_{H}\left(n_{0}+n\right)
$$

and from eq. (5.1.12) $m=\frac{n_{0} m_{H}}{\sqrt{1-\frac{V^{2}}{c^{2}}}}$, where $m$ is a relativistic

mass of the moving body, therefore we get

$$
m=m_{H}\left(n_{0}+n\right)
$$

and by solving eq. (5.1.11) in terms of the velocity, we get

$$
V= \pm \sqrt{\frac{n^{2}+2 n n_{0}}{\left(n+n_{0}\right)^{2}}} c
$$

Now, when a body absorbs energy with frequency $v$ so the velocity of this body in terms of time is given by substituting the value of $n$ from eq. (5.1.1) in the eq. (5.1.14), we get

$$
V= \pm\left[\frac{(v t)^{2}+2(v t) n_{0}}{\left[(v t)+n_{0}\right]^{2}}\right]^{\frac{1}{2}} c
$$

and also we can express eq. (5.1.13) in terms of time, where we get

$$
m=m_{H}\left(n_{0}+v t\right)
$$

The relativistic momentum [21] for a body moving with constant velocity $V$ is given by the relation

$$
P=m V
$$

where $\mathrm{P}$ is the momentum, and from eqs. (5.1.15) and (5.1.16) we can get the momentum in terms of time, where we have

$$
P= \pm m_{H} c \sqrt{(v t)^{2}+2(v t) n_{0}}
$$

Newton's second law of motion is given by the relation

$$
F=\frac{d P}{d t}
$$

where $F$ is the force. and by deriving eq. (5.1.17) with respect of time, we get

$$
F= \pm m_{H} c\left[\frac{v^{2} t+v n_{0}}{\sqrt{(v t)^{2}+2(v t) n_{0}}}\right]
$$

and by multiplying equation (5.1.18) by $\frac{c}{c}$ we get

$$
F= \pm m_{H} c^{2}\left[\frac{(v t)+n_{0}}{\sqrt{(v t)^{2}+2(v t) n_{0}}}\right] \frac{1}{c}
$$

and from eq. (5.1.15) we have $\frac{1}{V}=\left[\frac{(v t)+n_{0}}{\sqrt{(v t)^{2}+2(v t) n_{0}}}\right] \frac{1}{c}$, and from eq. (5.1.9), we have $H=m_{H} c^{2}$. Now by substituting these values in (5.1.19) we get 


$$
F= \pm \frac{H v}{V}
$$

Equation (5.1.20) expresses about the affected force on a body, when the body changes its velocity from zero to $V$, when it absorbs a photon with frequency $v$, and we find the dimension of eq. (5.1.20) is $M L T^{-2}$ which means force, and by taking the positive value of eq. (5.1.20), we get

$$
F=\frac{H v}{V}
$$

Equation (5.1.21) agrees with the equation of describing the momentum of the photon in quantum theory, where the momentum of a photon is $P=\frac{h v}{c}$, and this agrees with the core of wave-particle duality.

Now suppose a body starts at rest $(V=0)$, and after it absorbed a photon with frequency $v_{1}$, its velocity became $V_{1}$, and according to the eq. (5.1.21), the force affected on the body is $F_{1}$, where $F_{1}=\frac{H v_{1}}{V_{1}}$ and then after it absorbed another photon with frequency $v_{2}$ therefore the body should move with a total velocity $V$ (because of the absorption the two photons $v_{1}$ and $\left.v_{2}\right)$. So the total force affected on the body is $F=\frac{H\left(v_{1}+v_{2}\right)}{V}$. The affected force on the body as a result of the absorption of the second photon $v_{2}$ is $F_{2}$ where

$$
F_{2}=F-F_{1}
$$

Equation (5.1.22) is very important for understanding the equivalence principle of my theory.

\section{6- The ReLATIVISTIC Quantized InerTial ForCe, THE RELATIVISTIC QUANTIZED GRAVITATIONAL FORCE}

\subsection{The relativistic Quantized Inertail Force}

As we know from the Quantum Theory that the energy is photons having a rest mass equals to zero $[1,2,5,12,14]$. We can express the photon energy by the relation

$$
E=h v
$$

Where $E$ is the photon energy, $h$ is plank's constant and $v$ is the wave frequency $[5,6]$. And from the equivalence of mass and energy, we can get the equivalent mass $m$ to a photon having energy $E$ as

$$
m=\frac{h v}{c^{2}}
$$

Now suppose a train moving with constant velocity $V$ ( remember we use in this section and in the others V-capital letter to define the velocity, and $v$ to define to the frequency), as we have from the special relativity theory of Einstein the clock motion of the moving train should be slower than the clock motion of the earth observer for the observer of the earth surface which that agrees with my interpretation to the Lorentz transformation, whereas if the earth observer measured the time interval $\Delta t$ via his earth clock, then he will measure the time interval $\Delta t^{\prime}$ via the clock of the moving train, where $\Delta t^{\prime}=\sqrt{1-\frac{V^{2}}{c^{2}}} \Delta t$ [16]. And the wave frequency is defined as the cycle number in a unit of time, and subsequently the wave frequency which exists on the earth surface according to the earth observer is given by the relation

$$
v=\frac{1}{\Delta t_{0}}
$$

And now if this wave entered inside the moving train, then, the wave frequency becomes $v^{\prime}$ according to the earth observer, where

$$
v^{\prime}=\frac{1}{\Delta t}=\frac{\sqrt{1-\frac{V^{2}}{c^{2}}}}{\Delta t_{0}}
$$

And from that we get

$$
v^{\prime}=\sqrt{1-\frac{V^{2}}{c^{2}}} v
$$

Equation (6.1.4) indicates that the wave frequency inside the moving train should be less than outside the train on the earth surface by the factor of $\sqrt{1-\frac{V^{2}}{c^{2}}}$. Subsequently the endured energy $E^{\prime}$ through this photon inside the moving train is given by

$$
E^{\prime}=h v^{\prime}=\sqrt{1-\frac{V^{2}}{c^{2}}} h v
$$

And from eq. (6.1.1), we get

$$
E^{\prime}=\sqrt{1-\frac{V^{2}}{c^{2}}} E
$$

Equation (6.1.5) represents the endured energy inside the frame of the moving train according to the reference frame of the earth surface in terms of the photon energy $E$. The difference of the endured energy $\Delta E$ of the moving train from its rest on the earth surface and its motion with constant velocity $V$ is given by the relation 


$$
\Delta E=E\left[1-\sqrt{1-\frac{V^{2}}{c^{2}}}\right]
$$

We have reached in the preceding section to a new formula for understanding the quantization of force, where the force acts on the body when its velocity changes from zero to $V$ is given by the relation $F=\frac{H v}{V}$.

Now suppose a stationary train on the earth surface and a rider is living inside it, Now if this train absorbs an energy of frequency $v$, then the speed of this train will change from 0 to $V$, thus, the affected force on this train is given by the relation $F=\frac{H v}{V}$ according to the stationary earth observer, in this case there is a force affected on the rider pushing him to the opposite direction of the train's speed change. This force is called "inertial force". Subsequently, according to this force the rider's speed should be changed also from 0 to $V_{r}$ locally, whereas in this case $V_{r}$ should be equal to $V$ (the speed of the train). We can get this change of the velocity of the train rider from 0 to $V_{r}$ under the affect of inertial force whereas $V_{r}$ should be equal to $V$ by applying the two conditions

1- $\quad$ The kinetic energy $E_{k}$ that is equivalent to the rider's speed $V_{r}$ is given as

$$
E_{k}=E_{0}\left(1-\gamma^{-1}\right)
$$

Where $\gamma^{-1}=\sqrt{1-\frac{V^{2}}{c^{2}}}$, and $E_{0}$ is the equivalent energy of the rider's rest mass, where $E_{0}=m_{0} c^{2}$. We can express the kinetic energy in the last equation in the terms of the number of $H$-energy, where we have

$$
n=n_{0}\left(1-\gamma^{-1}\right)
$$

Where $n$ is the number of $H$-energy which is equivalent to the kinetic energy, and $n_{0}$ is the number of $H$-particle or the number of the $H$-energy which equivalent to the rider's rest mass.

2- $\quad$ The endured rest mass inside the train in terms of the rider's rest mass is $m_{0}{ }^{\prime}$ given according to eq. (6.1.5), where we have

$$
m_{0}{ }^{\prime}=\gamma^{-1} m_{0}
$$

And we can express the last equation in terms of $H$-particle or $H$-energy, where we have

$$
n_{0}{ }^{\prime}=\gamma^{-1} n_{0}
$$

Where $n_{0}{ }^{\prime}$ is the number of $H$-particleor the number of $H$-energy which is equivalent to the endured rest mass, thus, from eq. (6.1.7) we can write the last equation as

$$
n_{0}{ }^{\prime}=n_{0}-n
$$

Now according to these two conditions, we can get the measured speed $V_{r}$ locally of a rider under the affect of the inertial force according to the observer inside the train by eq. (5.1.14), where we have

$$
V_{r}=\sqrt{\frac{n^{2}+2 n n_{0}{ }^{\prime}}{\left(n+n_{0}{ }^{\prime}\right)^{2}}} c=\sqrt{\frac{n^{2}+2 n\left(n_{0}-n\right)}{\left[n+\left(n_{0}-n\right)\right]^{2}}} c
$$

by substituting $n_{0}{ }^{\prime}=n_{0}-n$, we get

$$
V_{r}=\sqrt{\frac{2 n n_{0}-n^{2}}{n_{0}^{2}}} c=\sqrt{\frac{2 n}{n_{0}}-\frac{n^{2}}{n_{0}^{2}}} c
$$

And from eq. (6.1.7) we get

$$
V_{r}=\sqrt{\frac{2 n_{0}\left(1-\gamma^{-1}\right)}{n_{0}}-\frac{n_{0}^{2}\left(1-\gamma^{-1}\right)^{2}}{n_{0}^{2}}} c
$$

And from that we get

$$
V_{r}=\sqrt{1-\gamma^{-2}} c
$$

And by substituting the value of $\gamma^{-2}=1-\frac{V^{2}}{c^{2}}$ in the last equation we get

$$
V_{r}=V
$$

We get from eq. (6.1.9) that the change in the measurement of the train's rider speed under the effect of the inertial force is from 0 to $V$ locally and it is the same change of the train's speed, but it is in the opposite direction. Therefore we get the inertial force $F_{i}$ which acts on the train's rider locally, whereas from eq. (5.1.21) we have

$$
F_{i}=\frac{H v}{V}=\frac{H v_{0}\left(1-\gamma^{-1}\right)}{V}
$$

Now for an observer on the ground, if the change of the speed of the rider locally under the effect of the inertial force is from 0 to $V$, then for the observer on the ground the change of the speed of the rider is from 0 to $\gamma^{-1} V$ as from eq. (21) in my interpretation of the Lorentz transformation, thus the measured inertial force affected on the rider of the train for the observer on the ground is given as

$$
F_{i}^{\prime}=\frac{H v_{0}\left(1-\gamma^{-1}\right)}{\gamma^{-1} V}
$$

\subsection{The Relativistic Quantized Gravitational Force and the Quantized Gravitational Time Dilation}

The relativistic quantized inertial force locally is given according to the equation (6.1.10), where

$$
F_{i}=\frac{H v_{0}\left(1-\gamma^{-1}\right)}{V}
$$

Now, according to my equivalence principle, the inertial force is equivalent to gravitational force locally. That means when 
the velocity of the train changes from 0 to $\mathrm{V}$, then locally the velocity of the rider on the train will change from 0 to $\mathrm{V}$ also as a result of the inertial force. Thus by my equivalence principle we can use equation (6.1.10) for computing the gravitational force. Here locally we have

$$
V_{r}=V=V_{\text {escape }}
$$

given as in eq. (6.1.8).

Now if a body of rest mass $m_{0}$ is located at a gravitational field of big mass $\mathrm{M}$. Thus by my equivalence principle, the kinetic energy equivalent to the change of the velocity of the body locally from 0 when it is located at a distance equals to infinity from the big mass $\mathrm{M}$ to $V_{r}=V=V_{\text {escape }}$ at radius $\mathrm{r}$ from the center of the big mass $\mathrm{M}$ is ${ }^{r} E$ given by

$$
E=m_{0} c^{2}\left(1-\gamma^{-1}\right)
$$

Equation (6.2.1) indicates us according to my equivalence principle, apart of the rest mass of the free falling object in gravity will change to energy (photons), and these photons give the object the kinetic energy equivalent to the escape velocity locally.

Now if we consider this energy is equal to the gravitation potential energy, from that we get

$$
\frac{G M m_{0}}{r}=m_{0} c^{2}\left(1-\gamma^{-1}\right)
$$

$G$ is the gravitational constant

$M$ is the mass of the gravitational field

$m$ is the mass of the body

$r$ is the distance between the body and the mass $M$

Thus we can solve the equation above to get the Lorentz factor $\gamma^{-1}$ of gravity where

$$
\gamma^{-1}=1-\frac{G M}{c^{2} r}
$$

From that we can get the gravitational time dilation, whereas if clock 1 is located at a distance $r$ from the center of the ma: $\mathrm{M}$, thus the time that is measured by this clock is $\Delta t^{\prime}$ for $\mathrm{c}$ observer located at $\mathrm{r}=$ infinity compared to the time $\Delta t$, clock 2 located at $r=$ infinity from the center of mass $\Lambda$ whereas

$$
\Delta t^{\prime}=\gamma^{-1} \Delta t
$$

Thus

$$
\Delta t^{\prime}=\left[1-\frac{G M}{c^{2} r}\right] \Delta t
$$

Now if we consider $\gamma^{-1}=0$, then we can compute the radius that the big mass $M$ should be compressed to be transformed to a black hole. This radius is known as the Schwarzschild radius. Thus

$$
1-\frac{G M}{c^{2} r_{s}}=0
$$

Thus

$$
r_{s}=\frac{G M}{c^{2}}
$$

Whereas $r_{s}$ is the Schwarzschild radius [22].

Now we can compute $r_{s}$ for the earth where

$$
r_{s}=0.0044318 \mathrm{H}
$$

Schwarzschild's calculated gravitational radius according to GR differs from this result by a factor of 2 and is coincidently equal to the non-relativistic non-quantized escape expression velocity which depends on the Einstein's equivalence principle.

Now if we consider for the earth $\gamma^{-1}=1-\frac{G M}{C^{2} R}$, where $R$ is the radius of the earth, and $M$ is its mass. Thus by taking

$$
\begin{aligned}
& M=5.98 \times 10^{24} \mathrm{~kg} \\
& G=6.67 \times 10^{-11} \mathrm{~N} \mathrm{~m}^{2} \mathrm{~kg}^{-2} \\
& R=6.38 \times 10^{6} \mathrm{~m} \\
& C=3.0 \times 10^{8} \mathrm{~m} / \mathrm{s}
\end{aligned}
$$

Then $\gamma^{-1}=1-\left(6.95 \times 10^{-10}\right) \approx 0.99999999305353$ \&

From that we can get the gravitational time dilation of clock1 located on the earth surface comparing to clock2 located at infinity from the earth for an observer located at infinity as in equation (6.2.3) whereas

$$
\Delta t^{\prime}=0.9999999993053535 \Delta \mathrm{t}
$$

From that if clock2 registered one second for an observer at infinity, at this moment clock1 will register 0.9999999993053535 second for an observer located at infinity.

In this case the difference of time is $6.946646 \times 10^{-10}$ second. But for an observer located on the earth surface looking at clock 2 located at infinity, he will get that clock 2 is moving in a similar rate of his clock on the earth surface, where clock 2 is not moving in a slower rate than of his earth clock according to my new interpretation to the Lorentz transformation equations, where according to my transformation I refuse the reciprocity principle in Einstein's SRT. This leads the observer on the earth surface to measure the possibility of faster than light (review fig. (2) and how the possibility of measuring faster than light in my theory).

In the equivalence principle of my theory, the time dilation of the clock on the earth surface is produced as the clock on the ground is moving with speed equals to the escape velocity, which is agreed completely with the core of the The PoundRebka experiment [44]. Proponents of the theory of General Relativity offer three different conflicting explanations of these results that are said to be equivalent to each other and therefore all equally correct. All make the claim that the results of the Pound-Rebka Experiment are "proof" of the 
Equivalence Principle even though nothing in these measurements suggests any need for the Equivalence Principle. Also according to my interpretation to the Lorentz transformation equation it is possible measuring of a speed of light to be faster than c. The possibility of superluminal photon propagation in gravitational fields is one of the most remarkable predictions of quantum field theory in curved spacetime. It appears that real photons propagating in a variety of background spacetimes may, depending on their direction and polarisation, travel with speeds exceeding the normal speed of light c. This phenomenon was discovered by Drummond and Hathrell in 1980 [31]. It is a quantum effect induced by vacuum polarisation and implies that the Principle of Equivalence in GR is violated in interacting quantum field theories such as QED. But according to my interpretation to the Lorentz transformation equations and my equivalence principle this problem is solved.

The relativistic escape velocity locally of a body to be free from the earth gravity is given by equation (6.1.8), where $V_{\text {escape }}=\sqrt{1-\gamma^{-2}} c$. Thus the relativistic escape velocity locally on the earth is $V_{\text {escape }}=11182 \mathrm{~m} / \mathrm{s}$. The force that is exerted on a body of mass $1 \mathrm{~kg}$ to move from 0 to $V_{\text {escape }}$ locally is given by the equation (6.1.10) where $F=5590.98$ Newt ons. This result is half the classical result. That refers to the relativistic quantized derivation of the momentum in my model [5,6] which leads to the group velocity is half the classical velocity. From here it comes the importance of factor 2 that is existed in the Schwarzschild solution in the Lorentz factor, where in the case of making an approximation from the quantized force to the classical force or classical acceleration in the case of weak gravitation, then, we must multiply it by the factor of 2 in order to be equal to the classical force or classical acceleration. And this factor of 2 makes the confusion for physicists in all attempts to unify between QFT and GR.

For an observer located at infinity from the earth, the measured escape velocity for an object located on earth is less than the escape velocity locally by a factor of $\gamma^{-1}$ as in eq. (21), where it is equal to

$$
V_{\text {escape }}^{\prime}=\gamma^{-1} \sqrt{1-\gamma^{-2}} c
$$

In this case and according to eq. (6.2.6), there must be a measured red shift in the kinetic energy of an object to be free from gravity for an observer located at infinity. This red shift was noticed in the Pound-Rebka experiment [44].

Equation (6.2.6) can be derived by the Schwarzschild geometry in GR in the case of freely falling particle in weak gravity. Where from (6.2.2), we can get

$$
\gamma^{-1}=\sqrt{\left(1-\frac{G M}{c^{2} r}\right)^{2}}=\sqrt{1-\frac{2 G M}{c^{2} r}+\frac{G^{2} M^{2}}{c^{4} r^{2}}}
$$

And in the case of weak gravitational field we have

$$
\frac{G^{2} M^{2}}{c^{4} r^{2}}<<\frac{2 G M}{c^{2} r}
$$

Thus in the case of weak gravitational we get

$$
\gamma^{-1} \approx \sqrt{1-\frac{2 G M}{c^{2} r}}
$$

Which is the Lorentz factor derived by Schwarzschild solution in GR. Thus the Lorentz factor derived by my theory is equal to the Lorentz factor of Schwarzschild solution in the case of weak gravitational field.

Thus eq. (6.2.6) will be approximated in case of weak gravitational field as

$$
V_{\text {escape }}^{\prime} \approx \sqrt{1-\frac{2 G M}{c^{2} r}} \sqrt{\frac{2 G M}{r}} \approx\left(1-\frac{G M}{c^{2} r}\right) \sqrt{\frac{2 G M}{r}}
$$

According to the Schwarzschild geometry in GR of freely falling object in gravity we get

$$
V_{\text {escape }}^{\prime}=\left(1-\frac{2 G M}{c^{2} r}\right) \sqrt{\frac{2 G M}{r}}
$$

We have seen the difference between eqs. (6.2.7) and (6.2.8) is the factor of 2 in the Lorentz factor. This is produced by the equivalence principle that adopted by Einstein in his GR depending on the classical velocity equivalent to classical kinetic energy and classical acceleration, but in the equivalence principle that adopted by my theory is depending on the velocity equivalent to the relativistic kinetic energy and quantum theory, and the concept of acceleration in my theory is equivalent to the vacuum fluctuations.

According to my equivalence principle and the quantization of GR, the change in the speed of light when passing through gravity or any particle which owns rest mass greater than zero (freely falling particle in gravity) the change in a photon's or particle's velocity depends not on the strength of the gravitational field, but on the gravitational potential itself as in eq. (6.2.2). Whereas for the light beam passing through gravity, the measured speed of light globally given according to eq. (20), and by my equivalence principle according to eq. (6.2.2) we get

$$
c^{\prime}=\gamma^{-1} c=\left(1-\frac{G M}{c^{2} r}\right) c
$$

Franson [32] calculated that, treating light as a quantum object, the change in a photon's velocity depends not on the strength of the gravitational field, but on the gravitational potential itself. However, this leads to a violation of Einstein's equivalence principle - that gravity and acceleration are indistinguishable - because, in a gravitational field, the gravitational potential is created along with mass, whereas in a frame of reference accelerating in free fall, it is not. Therefore, one could distinguish gravity from acceleration by whether a photon slows down or not when it undergoes particle-antiparticle creation. As we have seen previously, Frason's calculations is agreed completely with my equivalence principle. 
Suppose a particle fell in a Schwarzschild radius, thus according to my equivalence principle, that is equivalent as the velocity of the moving train changes from 0 to $c$ the speed of the light in vacuum, and thus the velocity of the rider will change locally from 0 to c also. Thus, the applied force $F_{g}$ on the particle locally in the Schwarzschild radius is given according to eq. (6.1.10) where

$$
F_{g}=\frac{H v_{0}}{c}
$$

Here $v_{0}$ is the equivalent frequency of the rest mass energy of the particle, where in the Schwarzschild radius $\gamma^{-1}=0$, and thus according to eq. (6.2.1) all the rest mass of the particle will change to photons, and then the particle will move in the speed of light locally and then the applied gravitational force locally on the particle equals to the force of light as in the equation above.

Now, for an observer located at infinity or on earth surface from the black hole, and from eqs. (20) \&(21); if the inertial force let the velocity of the rider of the train to change from 0 to $c$ locally as a result of the velocity of the train changes from 0 to $c$, then for the observer on the ground, the change of the velocity of the rider under the effect of the inertial force will be from 0 to $\sqrt{1-\frac{V^{2}}{c^{2}}} c=0$, where $V=c$. This is equivalent to for an observer located at infinity or on earth from the black, and a particle fell in the back hole at the Schwarzschild radius, then according to my equivalence principle the velocity of the particle will change from 0 when the particle is located at infinity to $\gamma^{-1} c=0$ when the particle fell at the Schwarzschild radius relative to the observer located at infinity or on earth.

Thus from eq. (6.1.11), the applied gravitational force on the particle fell at the Schwarzschild radius is equal to infinity for an observer located at infinity or on earth. The Schwarzschild radius in my theory is given according to eq. (6.2.4).

Finally, as we have seen in my proposed quantization of General relativity of Einstein, There is no graviton! photon mediates gravitation!

\section{ACKNOWLEDGEMENT}

I would like to thank my wife who helped me by her patient to perform this research, and also my children, Rose, Shahid, Majd and Taleb. They grew up now! but without seeing them growing up in front of my eyes. My love to my family, and the pain of traveling without them, that helped me to understand what is the meaning of space and time in relativity and what is the meaning of past, present and the future. Also, I would like to thank the great country, Switzerland, by using their trains, and living the Einstein's dream that opened my mind to understand Relativity more when I was riding the Einstein's train of Relativity. Finally, great thanks to the Swiss
People, their smile always makes me optimistic among the painful moments.

\section{REFERENCES:}

[1] Heisenberg, W., "Physics and Phylosophy", (1958), Allien and Unwin.

[2] Pais, A., "Einstein and The Quantum theory", (1979),Rev. of Modern Physics, vol. 51, No. 4.

[3] Stapp, H. "The Copenhagen School Interpretation and the nature of spacetime", (1972), American Journal of Physics, 40, 1098.

[4] Roland, W. Clark, "Einstein: The Life and Time", (1979), Hodder Stoughton, London, Sydney, Auckland, Toronto.

[5] Robert, E., "Quantum Physics of Atoms, Melecules, Solids, Nuclei, and Particles", (1985), John Wiley and Sons, 2nd ed..

[6] Richard, L. Liboff, "Introductory Quantum Mechanics", (1992), AddisonWesley Publishing Company, Inc., 2nd ed..

[7] Einstein, A., "Autobiographical notes", in P. A. Schilpp, editor, Albert Einstein; Philosopher-Scientist.

[8] H. J., Pain, " The Physics of Vibrations and Waves", (1993),John Wiley \& Sons, 4 th ed..

[9] Jerry, B. Marion, Stephen, T. Thornton, "Classical Dynamics of Particles \& Systems", (1988), Harcourt Brace Jovanovich, 3rd ed..

[10] Henry, Semat, "Introduction to Atomic and Nuclear Physics", (1962), Holt Rinehart Winston, 4th ed..

[11] Popper, K. R., "Objective Knowledge", (1983), Oxford at the Clarendon Press.

[12] Bohm, D., "Causality and Chance in Modern Physics", (1984), Routledge and Kegan Paul.

[13] Heisenberg, W., "Physics and beyond", (1971), London Allen \& Unwin.

[14] W. Cropper, "The Quantum Pysics", (1970), Oxford.

[15] W. Rosser, "An Introduction to the Theory of Relativity", (1972), Butterworth

[16] George T. Gillies. "The Newtonian gravitational constant: recent measurements and related studies", (1997), Reports on Progress in Physics, 60:151-225

[17] C. W. Misner, K. S. Thorne and J. A. Wheeler, Gravitation, W. H. Freeman and Company, New York (1973).

[18] Hans Ohanian and Remo Ruffini "Gravitation and Spacetime", (1994), 2nd edition, Norton, New York.

[19] Odenwald, S. \& Fienberg, RT.; "Galaxy Redshifts Reconsidered", (1993), in Sky \& Telescope Feb. 2003; pp31-35.

[20] Binney, James, and Michael Merrifeld, "Galactic Astronomy", (1998), Princeton University Press.

[21] Taylor, Edwin F.; Wheeler, John Archibald, "Spacetime Physics: Introduction to Special Relativity" (1992). (2nd ed.).

[22] Schwartzchild B "Probing the early Universe with quasar light", (1987), Physics Today 40; Nov. 17-20.

[23] Adler, R., Bazin M., and Schiffer, M., "Introduction to General Relativity", (1975), 2d ed., McGraw-Hill, New York.

[24] Carlip, S., "QuantumGravity in 2+1 Dimensions" (1998), Cambridge University Press, Cambridge.

[25] AlMosallami, Azzam. "Time Contraction: The Possibility of Faster Than Light Without Violation of Lorentz Transformation or Causality and the Vacuum Energy Dependent.” IJSER 5, no. 4 (April 20, 2014): 128-142. doi:10.14299/ijser.2014.04.001.

[26] A., AlMosallami, "Time Contraction: The Possibility of Faster Than Light Without Violation of Lorentz Transformation or Causality and the Vacuum Energy Dependent.” Int. J. Modern Theo. Physics, 2014, 3(1): 44-73.

[27] A. Almosallami, A Modified Special Relativity Theory in the Light of Breaking the Speed of Light, http://vixra.org/pdf/1111.0001v1.pdf.

[28] A. AlMosallami, "The Exact Solution of The Pioneer Anomaly According to The General Theory of Relativity and The Hubble's Law", http://vixra.org/abs/1109.0058.

[29] A. Almosallami, The comalogical theory, http://www.gsjournal.net/old/physics/azzam3.pdf

[30] A. Almosallami, The Comalogical Theory, Toward a Science of Consciousness, Arizona, Tucson (2008).

[31] I.T. Drummond and S.J. Hathrell, Phys. Rev. D22 (1980) 343 
[32] J D Franson 2014 New J. Phys. 16065008 doi:10.1088/1367-2630/16/6/065008

[33] Phys. Rev. Lett. 111, 211603 (2013).

[34] Phys. Rev. Lett. 111, 211602 (2013).

[35] Marcel Urban, François Couchot, Xavier Sarazin, Arache Djannati- Atai. The quantum vacuum as the origin of the speed of light. The European Physical Journal D, 2013; 67 (3) DOI: 10.1140/epjd/e2013- 30578-7.

[36] Magueijo, João, "New varying speed of light theories", Reports on the Progress of Physics, 66 (2003) 2025.

[37] H. P. Robertson, Rev. Mod. Phys. 21, 378 (1949).

[38] Brown, Harvey R., Michelson, FitzGerald and Lorentz: the Origins of Relativity Revisited.

[39] Macrossan, Michael N. (1986), "A Note on Relativity Before Einstein", Brit. Journal Philos. Science 37: 232-34.

[40] A. Halpern (1988). 3000 Solved Problems in Physics. Schaum Series. Mc Graw Hill. p. 688. ISBN 978-0-07-025734-4.

[41] Relativity DeMystified, D. McMahon, Mc Graw Hill (USA), 2006, ISBN 007-145545-0.

[42] Michelson, Albert Abraham \& Morley, Edward Williams (1887). "On the Relative Motion of the Earth and the Luminiferous Ether". American Journal of Science 34: 333-345.

[43] A. Almosallami " Time Contraction: the Possibility of Faster Than Light Without Violation of Lorentz Transformation or Causality and the Vacuum Energy Dependent" http://vixra.org/abs/1401.0043.

[44] Pound, R. V.; Rebka Jr. G. A. (November 1, 1959). "Gravitational Red-Shift in Nuclear Resonance". Physical Review Letters 3 (9): 439-441. 